\title{
A derivation of the statistical characteristics of forest fires
}

Jianyi Lin (jianyi.lin@unimi.it)

Sergio Rinaldi (rinaldi@elet.polimi.it)

\section{Approved by}

Ulf Dieckmann

Leader, Evolution and Ecology Program

June 2010

Interim Reports on work of the International Institute for Applied Systems Analysis receive only limited review. Views or opinions expressed herein do not necessarily represent those of the Institute, its National Member Organizations, or other organizations supporting the work. 


\title{
A Derivation of the Statistical Characteristics of Forest Fires
}

\author{
Jianyi Lin* and Sergio Rinaldi ${ }^{\bullet}+$
}

December 20, 2008

* Department of Mathematics, University of Milan, Milano, Italy

- Department of Electronics and Information, Politecnico di Milano, Milano, Italy

- EEP, International Institute for Applied Systems Analysis, Laxenburg, Austria

+ corresponding author:

Dr. S. Rinaldi

DEI, Politecnico di Milano

Via Ponzio 34/5, 20133, Milano, Italy

phone +39 0223993563

fax +3902 23993412

e-mail rinaldi@elet.polimi.it

Abstract

The analysis of large data sets concerning fires in various forested areas of the world has pointed out that burned areas can often be described by different power-law distributions for small, medium and large fires and that a scaling law for the time intervals separating successive fires is fulfilled. The attempts of deriving such statistical laws from purely theoretical arguments have not been fully successful so far, most likely because important physical and/or biological factors controlling forest fires were not taken into account. By contrast, the two-layer spatially extended forest model we propose in this paper encapsulates the main characteristics of vegetational growth and fire ignition and propagation, and supports the empirically discovered statistical laws. Since the model is fully deterministic and spatially homogeneous, the emergence of the power and scaling laws does not seem to necessarily require meteorological randomness and geophysical heterogeneity, although these factors certainly amplify the chaoticity of the fires. Moreover, the analysis suggests that the existence of different power-laws for fires of various scale might be due to the two-layer structure of the forest which allows the formation of different kinds of fires, i.e. surface, crown, and mixed fires. 
Empirical evidence of forest fires characteristics

27 Forest fires have been observed for centuries all over the world, and huge

28 data sets are now of public domain. They usually contain long series of fire events identified by location, time of occurrence, and burned area.

30 Statistical analyses of these data sets have allowed various authors to

31 identify, on a purely empirical basis, general characteristics of forest 32 fires.

Malamud et al. (1998) and Ricotta et al. (1999) were the first to 34 perform statistics of the burned areas. They arrived to the same con35 clusion, namely that burned areas are distributed as a power law, rep36 resented by a straight line in log-log scale. This conclusion is actually 37 surprising, because the only graph reported in Ricotta et al. (1999) 38 clearly shows that the distributions of small, medium and large fires 39 are well approximated by different power laws, and the same, though 40 less pronounced, effect is detectable in the plots obtained by Malamud 41 et al. (1998). Most likely, this slightly distorted interpretation of the 42 results had two targets: find an agreement with the theoretical studies 43 available at that time on self-organized critical forest-fire models (see 44 next section), and support the idea that the knowledge of the occur45 rence frequency of small and medium fires can be used to quantify the 46 risk of large fires.

55 (Telesca et al. (2005); Lasaponara et al. (2005); Ricotta et al. (2006)). 
56 The main result is the discovery of a high degree of time-clusterization

even if the burned areas are not distributed as a power-law. This means that the occurrence of large events mimics the process of occurrence of smaller events, thus allowing one to model the scarce big fires on the basis of the abundant small fires. This is neatly pointed out in Corral et al. (2008) where the fire catalog for all Italy in the period 19982002 is used to estimate the probability density $D(\tau \mid s)$ of the time intervals $\tau$ separating two successive fires within the so-called class$s$ fires (i.e., fires with burned areas grater than or equal to $s$ ). The distributions estimated for each class (see the curves displayed in Fig. 2(a) reproduced from Corral et al. (2008)) can somehow be fitted with a power-law, but the exponent of the power-law (i.e., the negative slope of the curve) decreases with the increase of the minimum burned area $s$ characterizing the class. However, all these distributions practically collapse into a single function $F$, as shown in Fig. 2(b) (again extracted from Corral et al. (2008)), through the simple scale transformation $\tau \rightarrow R(s) \tau$ and $D(\tau \mid s) \rightarrow D(\tau \mid s) / R(s)$, where $R(s)$ is the rate of fire occurrence in class $s$ (defined as the mean number of fires per unit time with burned area greater than or equal to $s$ ). This interesting discovery, formally revealed by the relationship

$$
D(\tau \mid s)=R(s) F(R(s) \tau)
$$

allows one to conclude that forest fires fulfill a scaling law for the time intervals separating successive fires without necessarily displaying powerlaw distributions of the burned areas.

[Figure 2 about here.]

\section{Theoretical investigations on forest fire characteristics}

The attempts of deriving fire characteristics from purely theoretical arguments have been performed through two different classes of models.

The models of the first class, known as self-organized critical forest-fire (SOCFF) models, are probabilistic cellular automata defined over a square lattice with $L^{2}$ sites. In the first version (Bak et al. (1990)) each site is at each time step in one of three possible states: green 
(i.e., not burning) tree; red (i.e., burning) tree; absence of vegetation.

The transition rules are very simple: $(i)$ green trees become red if they are close to red trees and remain green otherwise; (ii) red trees die thus leaving the site empty; (iii) each empty site has a probability $p$ of becoming occupied by a green tree. Bak et al. (1990) state that their model is a self-organized critical model capable of showing how the solar energy absorbed continuously at low rate by vegetation can be randomly dissipated through rare and disruptive events (the fires). However, the agreement with real forests is, even qualitatively, rather poor because the model generated fires are always present (in the form of travelling fronts burning pieces of the boundaries of vegetational clusters).

The model proposed by Bak et al. (1990) is immediately criticized by Drossel and Schwabl (1992) who point out some of its critical aspects and introduce a second parameter $(f)$, called "lightning parameter" by means of which they modify rule $(i)$ saying that green trees not close to red ones have a probability $f$ to become red. This variation introduces a random exogenous mechanism of fire ignition and is essential for creating clusters of fires with areas distributed as power-laws. A number of variants of the SOCFF model are immediately proposed by various authors (see Clar et al. (1996) for a review). In particular, Drossel and Schwabl (1993) introduce a third parameter, called "tree immunity" in order to modify, once more, rule $(i)$ by saying that green trees have a certain probability of remaining such when they are close to red trees. Later (Song et al. (2001)) this variant is shown to give rise to distributions of burned areas that can be approximated with two power-laws, one for small-medium fires and one for large fires. A similar result is obtained by Schenk et al. (2000) by stressing the finite-size effects in SOCFF models.

In the second class of models, here called two-layer models, the forest is described by two sets of ordinary differential equations, one associated with the lower layer composed of bryophytes, herbs, shrubs or any mix of these plants and the other associated with the upper layer composed of plants and trees of various species. The growth of the two layers in the absence of fire is described in the standard continuous time 


$$
\begin{aligned}
& \dot{L}=r_{L} L\left(1-\frac{L}{K_{L}}\right)-\alpha L U \\
& \dot{U}=r_{U} U\left(1-\frac{U}{K_{U}}\right)
\end{aligned}
$$

where $r$ and $K$ indicate growth rate and carrying capacity and $\alpha L U$ is the surplus of mortality in the lower layer due to light interception caused by tree canopy. Thus, in the absence of fire, trees grow logistically toward the carrying capacity $K_{U}$, while plants of the lower layer tend toward $\left(1-\alpha K_{U} / r_{L}\right) K_{L}$. The validity and limitations of eq. (2) are discussed in Casagrandi and Rinaldi (1999), where realistic values of the five vegetational parameters $\left(r_{L}, r_{U}, K_{L}, K_{U}, \alpha\right)$ are also suggested.

As for the fire, there are two options. The first (Casagrandi and Rinaldi (1999)) is to add two extra-variables representing the burning (red) biomasses in the two layers and describe the propagation of the fire to the green biomasses $L$ and $U$ through suitable fire attack rates. This gives a model with four ordinary differential equations which is, however, a so-called slow-fast model because the green biomasses grow very slowly (typically over years), while the red ones become suddenly very high when the fire starts and then practically drop to zero after a very short time (typically a few days or weeks).

The second option (Maggi and Rinaldi (2006)) is to push the slowfast nature of the system to the extreme, by considering fires as devastating events capable of reducing instantaneously the green biomasses of finite amounts. This can be accomplished, without adding extra differential equations, by defining as shown in Fig. 3(a) the pre- and post-fire manifolds $X^{-}$and $X^{+}$and the map from $X^{-}$to $X^{+}$interpreting the impact of the fire.

[Figure 3 about here.]

The pre-fire manifold $X^{-}$in Fig. 3(a) is piece-wise linear and nonincreasing, and the set below the manifold is convex. The first property is obvious because less fuel originated from trees (i.e. less trees) is necessary for fire ignition if more fuel originated from bushes is available on the ground. The second property simply says that if $x^{\prime}=\left(B^{\prime}, T^{\prime}\right)$ and $x^{\prime \prime}=\left(B^{\prime \prime}, T^{\prime \prime}\right)$ are two states of the forest at which fire ignition is 

not possible (i.e. two points below the manifold $X^{-}$) no mix of these two states (i.e. no points of the segment connecting $x^{\prime}$ with $x^{\prime \prime}$ ) can give rise to fire ignition. A formal support of these two properties can be found in Maggi and Rinaldi (2006). The geometry of the pre-fire manifold allows one to sharply identify surface fires (vertical segment of $X^{-}$), crown fires (horizontal segment of $\mathcal{X}^{-}$) and mixed fires (oblique segment of $\left.X^{-}\right)$. By definition, surface fires do not involve the upper layer, so that the post-fire conditions are on the vertical segment characterized by $L^{+}=\lambda_{L} \rho_{L} K_{L}=\lambda_{L} L^{-}$. In other words, $\rho_{L}$ is, by definition, the portion of the lower layer carrying capacity $K_{L}$ at which surface fires occur and $\lambda_{L}$ is the portion of the lower layer biomass that survives to surface fire. Similarly, fires in the upper layer are characterized by a vertical instantaneous transition from $U^{-}=\rho_{U} K_{U}$ to $U^{+}=\lambda_{U} U^{-}$. The most extreme surface fire is represented by the transition $S^{-} \rightarrow S^{+}$, while the most extreme crown fire is represented by the transition $C^{-} \rightarrow C^{+}$. The assumption that mixed fires initiate on the segment $C^{-} S^{-}$implies, by continuity, that post-fire conditions are on a curve connecting $C^{+}$and $S^{+}$which, for simplicity, is identified with the linear segment $C^{+} S^{+}$.

Fire sequences can be easily obtained from the model, as shown in Fig. 3(b). Starting from a given initial condition, say point 0, one numerically integrates the differential eqs. (2) until the solution hits the pre-fire manifold $X^{-}$at point $1^{-}$. Then, using the map $X^{-} \rightarrow X^{+}$ one can determine the post-fire conditions, namely point $1^{+}$. Finally, the procedure is iterated and a series of fires $\left(2^{-} \rightarrow 2^{+}\right),\left(3^{-} \rightarrow 3^{+}\right), \ldots$ is obtained.

A detailed analysis of this minimal model (Maggi and Rinaldi (2006)) has shown that it is very flexible and can reproduce, by tuning its parameters, the fire regimes of savannas, boreal forests and Mediterranean forests. The dependence of the model behavior upon its numerous parameters has been thoroughly investigated in Dercole and Maggi (2005) and in Bizzarri et al. (2008). Moreover, long series of model generated fires have been statistically analyzed and the result is that the distributions of the total biomasses burned by fire events (i.e., $L^{-}+U^{-}-L^{+}-U^{+}$) can often be approximated by three power 
laws (see Fig. 5 in Maggi and Rinaldi (2006)). This result is somehow

similar to that shown in Fig. 1(b), where, however, the fire intensities are identified with burned areas.

It is worth noticing that in none of the above mentioned studies the models have been validated against the data collected on a specific forest site. This is perfectly in line with the aim of the studies, which was to show that the models could produce fire regimes similar to those qualitatively observed in various biomes of the world.

\section{Analysis of a spatially extended two-layer forest fire model}

The models reviewed in the previous section are definitely poor and over-simplified from a biological point of view even if they support to a certain extent some of the characteristics of forest fires emerging from field data. SOCFF models reduce the growth of vegetation to a sort of unrealistic ballet of trees born in empty sites and then burned by lightning, without giving any role to important physical factors such as quantity of dead biomass on the ground or age of the plants which are known to control the ignition of a fire and its propagation (Viegas (1998)). By contrast, two-layer models are simply inappropriate for describing properties concerning burned areas because they do not explicitly contain space.

We therefore focus on a promising mix of the above models by spatially extending on a square lattice with $L^{2}$ sites, the two-layer forestfire model. Thus, eq. (2) holds at each site, characterized however by a different standing state $(L, U)$, and when the biomasses in one site reach the pre-fire manifold $X^{-}$, a fire is ignited in that site of the forest and the biomasses of that site are reduced in accordance with the map described in Fig. 3(a). Moreover, the fire propagates to neighboring sites provided the vegetation in those sites is almost ready to burn, i.e. provided the biomasses $(L, U)$ are $\varepsilon$-close to the pre-fire manifold $X^{-}$. In order to simplify the dynamics we assume, in accordance with Drossel and Schwabl (1992), that the propagation is a sort of instantaneous avalanche, since the time in which a forest cluster burns down is much shorter than the time in which a tree grows. This means that when the pre-fire manifold is reached at one site, the fire instan- 
taneously propagates to an entire forest cluster delimited by sites in

which the biomasses $(L, U)$ are at least $\varepsilon$-far from the pre-fire manifold $X^{-}$. Thus, the area burned by fire can be measured by the number of sites in the cluster.

Long simulations of the model allow one to generate long time series of fires with associated burned areas and times of occurrence. Since simulations involve time-discretization, it can happen (very rarely however) that two fires occur at the same time. In these cases one of the two fires is simply delayed of one time step.

In order to avoid finite-size effects we have been forced to work with large lattices and this is why, in order to keep computational effort under control, we have selected the model described in Maggi and Rinaldi (2006) which involves $2 L^{2}$ differential equations, i.e. one half of those that would be required by the model proposed in Casagrandi and Rinaldi (1999). Simulations must be very long because transients toward attractors of the extended forest model can be extremely long, in particular when the local dynamics, i.e. the dynamics of a single isolated site, are chaotic (see Fig. 4 which shows that a reliable estimate of the mean and standard deviation of the burned areas is obtained only after three hundred thousand years!).

[Figure 4 about here.]

Despite these computational difficulties, we have been able to perform reliable statistics of the burned areas and of the time of occurrence of the fires for different values of the parameters of the model. In particular, we have varied the parameter $\varepsilon$ that controls the tendency of the fire to penetrate into parts of the forest which are not yet ready to burn. Obviously, this parameter depends upon the dominant species present and can therefore vary remarkably in particular at continental scale. Higher values of $\varepsilon$ indicate lower resistance to fire propagation, i.e. lower tree immunity, as defined in Drossel and Schwabl (1993), Albano (1995) and Song et al. (2001) in their studies on SOCFF models. Higher values of $\varepsilon$ should therefore facilitate the occurrence of larger fires and this is, indeed, what we have systematically found with our simulations, as shown in Fig. 5 obtained for parameter values in the range suggested in Maggi and Rinaldi (2006) for Mediterranean forests. 
Figure 5 shows that the three basic types of distributions identified through empirical studies (see Fig. 1) can be produced by our model by varying the control parameter $\varepsilon$. Another interesting property of our model is that large fires, which are associated with the steepest slopes of the distributions of the burned areas, are mixed fires, while a relevant percentage of the small fires are surface fires. In other words, the results suggest that the existence of different slopes in the distributions of the burned areas might be due to the existence of differently structured fires. This has also been suggested in Schenk et al. (2000) but with totally different and less biologically based arguments.

Finally, long series of model generated fires have allowed us to estimate the probability density $D(\tau \mid s)$ of the time intervals $\tau$ separating successive fires with burned areas greater than or equal to $s$. A typical result of this analysis is shown in Fig. 6(a) which compares favourably with Fig. 2(a).

[Figure 6 about here.]

This means that our model captures also the processes that control the times of occurrence of the fires and not only the mechanism regulating the severity of the fires, i.e. the burned areas. But the qualitative agreement of our model with the empirical evidence goes even further. In fact, the similarity of Fig. 2(b) with Fig. 6(b), obtained through simulation, proves that the model is endowed with the scaling law (1) discovered on purely empirical grounds (Corral et al. (2008)).

\section{Concluding remarks}

We have shown that all statistical properties of forest fires discovered in the last decade through the analysis of available data can be derived from a biological based model in which the three phases of vegetational growth, fire ignition and fire propagation are clearly identified. In such a model the forest extends over a square lattice of $L^{2}$ sites and is composed of a lower and an upper layer. The two layers grow logistically, but the upper one reduces the light available to the lower one, thus damaging its growth. Fires are devastating instantaneous events that 
occur only when the mix of biomasses of the two layers reach particular values. The rationale for this assumption is as follows. We know (see, for example, Viegas (1998)) that fire ignition in a forest is possible only if dead biomass on the ground is above a certain threshold, but since the biochemical processes regulating the mineralization of dead biomass are relatively fast with respect to plant growth (Esser et al. (1982); Seastedt (1988)) it can be reasonably assumed that the rate of mineralization (proportional to the amount of dead biomass) equals the inflow rate of new necromass, which, in turn, is proportional to the standing biomass in the two layers. Thus, in conclusion, the biomasses of the two layers are appropriate indicators of fuel on the ground, so that fire ignition is possible only at sites where the standing biomasses reach specific conditions (called pre-fire conditions). When the fire is ignited at one site, it immediately propagates to the neighbouring sites if these are $\varepsilon$-close to their pre-fire conditions and this process is repeated in an avalanche like manner and stops only when the burning cluster is delimited by sites which are $\varepsilon$-far to their pre-fire conditions.

The combination of these slow and fast processes determines the behavior of the whole forest model which for parameter values in the ranges suggested in Maggi and Rinaldi (2006) for Mediterranean forests turns out to be chaotic. In other words, the slow and continuous growth of the two vegetational layers is punctuated by fires which occur in an apparently random way in space and time and has statistical properties consistent with those discovered empirically.

It is important to remark that the model proposed in this paper is nothing but the extension to a network of sites of the minimal model proposed in Maggi and Rinaldi (2006) for a single site. In other words, the model is still a minimal model that, as such, can not be calibrated for performing real time fire predictions in any specific forest, but rather be used to characterize and classify the fire regimes of large classes of forests.

It is also interesting to remark that the model is fully deterministic and spatially homogeneous, so that the emergence of the above statistical properties does not seem to be necessarily related with the randomness of meteorological conditions (soil moisture, wind speed, ...) 
or with geophysical heterogeneity. However, in accordance with Bessie and Johnson (1995) and Minnich and Chou (1997), we firmly believe that meteorological randomness and geophysical heterogeneity should amplify the chaoticity generated by the deterministic mechanisms of growth, ignition and propagation we have considered. Checking if this is true could be an interesting point for further investigation, in particular for assessing the impact of environmental change on fire regimes. But certainly more interesting would be to try to explain with the model important regional characteristics of fire regimes that have been discovered from data. For example, the east to west gradient of the slopes of the power-law distribution across US (Malamud et al. (2005)), might

be a consequence of a similar gradient in some of the parameters of the model, that control the slopes of the distributions.

\section{References}

Albano, E. V., 1995. Spreading analysis and finite-size scaling study of the critical behavior of a forest fire model with immune trees. Physica A $216,213-226$.

Bak, P., Chen, K., Tang, C., 1990. A forest-fire model and some thoughts on turbulence. Phys. Lett. A 147, 297-300.

Bessie, W. C., Johnson, E. A., 1995. The relative importance of fuels and weather on fire behavior in subalpine forests. Ecology 76 (3), $747-762$.

Bizzarri, F., Storace, M., Colombo, A., 2008. Bifurcation analysis of an impact model for forest fire prediction. Int. J. Bifurcat. Chaos 18 (8), $2275-2288$.

Casagrandi, R., Rinaldi, S., 1999. A Minimal Model for Forest Fire Regimes. Am. Nat. 153 (5), 527-539.

Clar, S., Drossel, B., Schwabl, F., 1996. Forest fires and other examples of self-organized criticality. J. Phys. Condens. Matter 8 (37), 68036824 .

Corral, A., Telesca, L., Lasaponara, R., 2008. Scaling and correlations 
in the dynamics of forest-fire occurrence. Phys. Rev. E 77, 016101(17).

Dercole, F., Maggi, S., 2005. Detection and continuation of a border collision bifurcation in a forest fire model. Appl. Math. Comput. 168, 623-635.

Drossel, B., Schwabl, F., 1992. Self-organized critical forest-fire model. Phys. Rev. Lett. 69 (11), 1629-1632.

Drossel, B., Schwabl, F., 1993. Forest-fire model with immune trees. Physica A 199 (2), 183-197.

Esser, G., Aselmann, I., Lieth, H., 1982. Modelling the Carbon Reservoir in the System Compartment "Litter". Mitt. Geol.-Paläont. Inst. Univ. Hamburg 52, 39-58.

Lasaponara, R., Santulli, A., Telesca, L., 2005. Time-clustering analysis of forest-fire sequences in southern Italy. Chaos Soliton. Fract. 24, 139-149.

Maggi, S., Rinaldi, S., 2006. A second-order impact model for forest fire regimes. Theor. Popul. Biol. 70, 174-182.

Malamud, B. D., Millington, J. D. A., Perry, G. L. W., 2005. Characterizing wildfire regimes in the United States. Proc. Nat. Acad. Sci. U.S.A. 102 (13), 4694-4699.

Malamud, B. D., Morein, G., Turcotte, D. L., 1998. Forest Fires: An Example of Self-Organized Critical Behavior. Science 281, 1840-1841.

Minnich, R. A., Chou, Y. H., 1997. Wildland Fire Patch Dynamics in the Chaparral of Southern California and Northern Baja California. Int. J. Wildland Fire 7 (3), 221-248.

Reed, W. J., McKelvey, K. S., 2002. Power-law behaviour and parametric models for the size-distribution of forest fires. Ecol. Model. 150, 239-254.

Ricotta, C., 2003. Fractal size distributions of wildfires in hierarchical landscapes: Natura facit saltus? Comm. Theor. Biol. 8, 93-101. 
Ricotta, C., Arianoutsou, M., Díaz-Delgado, R., Duguy, B., Lloret, F., Maroudi, E., Mazzoleni, S., Moreno, J. M., Rambal, S., Vallejo, R., Vázquez, A., 2001. Self-organized criticality of wildfires ecologically revisited. Ecol. Model. 141, 307-311.

Ricotta, C., Avena, G., Marchetti, M., 1999. The flaming sandpile: self-organized criticality and wildfires. Ecol. Model. 119, 73-77.

Ricotta, C., Micozzi, L., Bellelli, M., Mazzoleni, S., 2006. Characterizing self-similar temporal clustering of wildfires in the Cilento National Park (Southern Italy). Ecol. Model. 197, 512-515.

Schenk, K., Drossel, B., Schwabl, F., 2000. Finite-size effects in the selforganized critical forest-fire model. Eur. Phys. J. B 15 (1), 177-185.

Seastedt, T., 1988. Mass, Nitrogen, and Phosphorus Dynamics in Foliage and Root Detritus of Tallgrass Prairie. Ecology 69 (1), 59-65.

Song, W., Fan, W., Wang, B., Zhou, J., 2001. Self-organized criticality of forest fire in China. Ecol. Model. 145, 61-68.

Telesca, L., Amatulli, G., Lasaponara, R., Lovallo, M., Santulli, A., 2005. Time-scaling properties in forest-fire sequences observed in Gargano area (southern Italy). Ecol. Model. 185, 531-544.

Viegas, D. X., 1998. Forest Fire Propagation. Phil. Trans. R. Soc. Lond. A 356 (1748), 2907-2928. 
1 Three examples of cumulative distributions of burned areas obtained from data: (a) Clearwater National Forest (US) redrawn from Reed and McKelvey (2002); (b) Gargano (Italy) redrawn from Telesca et al. (2005); (c) Venaco (Corse, France) redrawn from Ricotta et al. (2001). The distribution in (a) cannot be approximated with a power law, while the distributions in (b) and (c) are approximated with three and two power laws, respectively. . . . . . . . . . . .

2 Results of the analysis of the time intervals $\tau$ separating successive fires in Italy (redrawn from Corral et al. (2008)). (a) Probability densities $D(\tau \mid s)$ for different minimum burned areas $s$. (b) The previous densities after rescaling by the mean fire rate $R(s)$ (notice that the rescaling yields dimensionless axes). . . . . . . . . . . . . . . .

3 Two-layer model behavior. (a) The pre- and post-fire manifolds $X^{-}$and $X^{+}$; the dotted lines with double arrows are instantaneous transitions from $X^{-}$to $X^{+}$due to a fire; horizontal (vertical) lines correspond to surface (crown) fires; oblique lines starting from the segment $C^{-} S^{-}$of $X^{-}$correspond to mixed fires. (b) State portrait of the model; continuous lines with a single arrow represent the growing phase of the forest and are described by eq. (2). . . . . . . . . . . .

4 Estimate $\hat{\mu}$ and $\hat{\sigma}$ of the mean and standard deviation of the burned areas as a function of the observation time for the model with $\varepsilon=0.08, r_{L}=3 / 8, r_{U}=1 / 16, K_{L}=K_{U}=$ $1, \alpha=129 / 800, \rho_{L}=0.85, \rho_{U}=14 / 15, \sigma_{L}=0.6, \sigma_{U}=$ $0.35, \lambda_{L}=\lambda_{U}=10^{-4} \ldots \ldots \ldots \ldots \ldots \ldots$

$5 \quad$ Three examples of cumulative distributions $P(s)=\operatorname{prob}[$ burned area $\geq$ $s$ ] obtained from the model for different parameter values: (a): $\varepsilon=0.06 ;(\mathrm{b}): \varepsilon=0.07 ;$ (c): $\varepsilon=0.08$. Other parameter values as specified in the caption of Fig. 4. . . . . . . .

6 Results of the analysis of the time intervals $\tau$ separating successive fires generated by the model with parameter values as specified in the caption of Fig. 4. (a) Probability densities $D(\tau \mid s)$ for minimum burned areas $s$. (b) The previous densities after rescaling by the mean fire rate $R(s)$. . . . . 

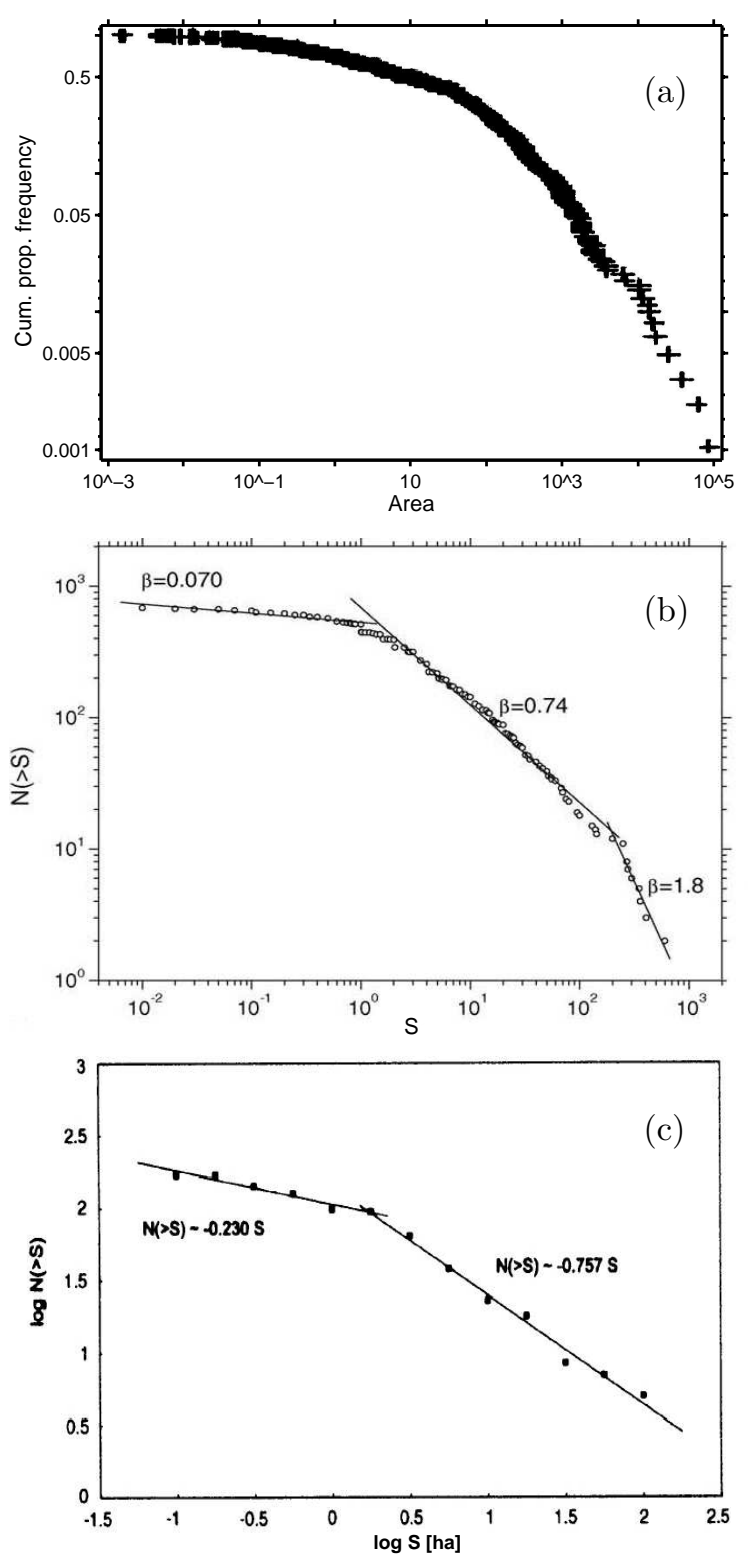

Figure 1: Three examples of cumulative distributions of burned areas obtained from data: (a) Clearwater National Forest (US) redrawn from Reed and McKelvey (2002); (b) Gargano (Italy) redrawn from Telesca et al. (2005); (c) Venaco (Corse, France) redrawn from Ricotta et al. (2001). The distribution in (a) cannot be approximated with a power law, while the distributions in (b) and (c) are approximated with three and two power laws, respectively. 

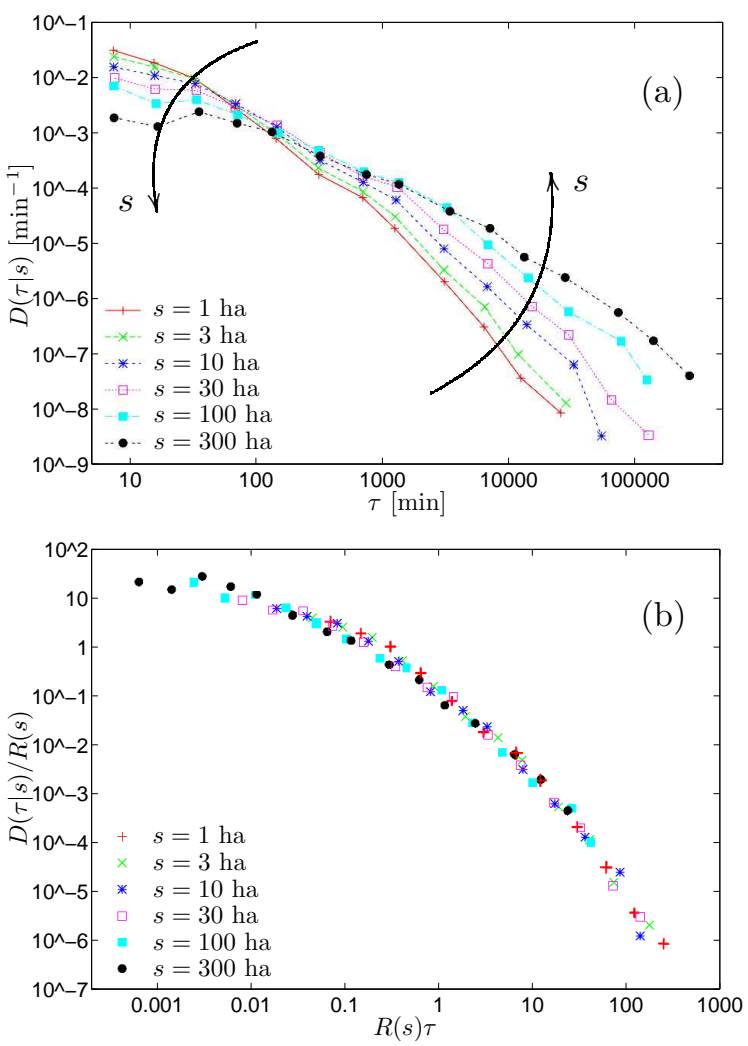

Figure 2: Results of the analysis of the time intervals $\tau$ separating successive fires in Italy (redrawn from Corral et al. (2008)). (a) Probability densities $D(\tau \mid s)$ for different minimum burned areas $s$. (b) The previous densities after rescaling by the mean fire rate $R(s)$ (notice that the rescaling yields dimensionless axes). 
(a)

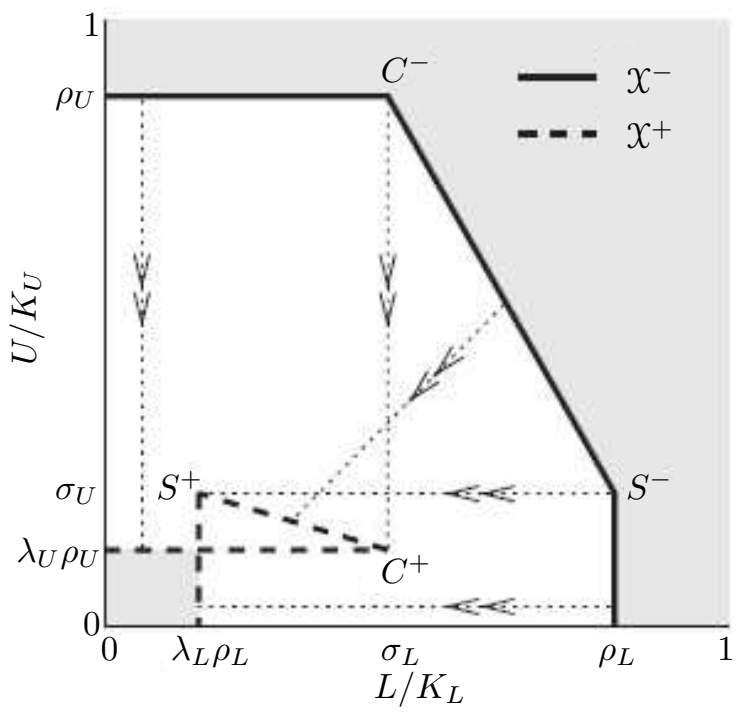

(b)

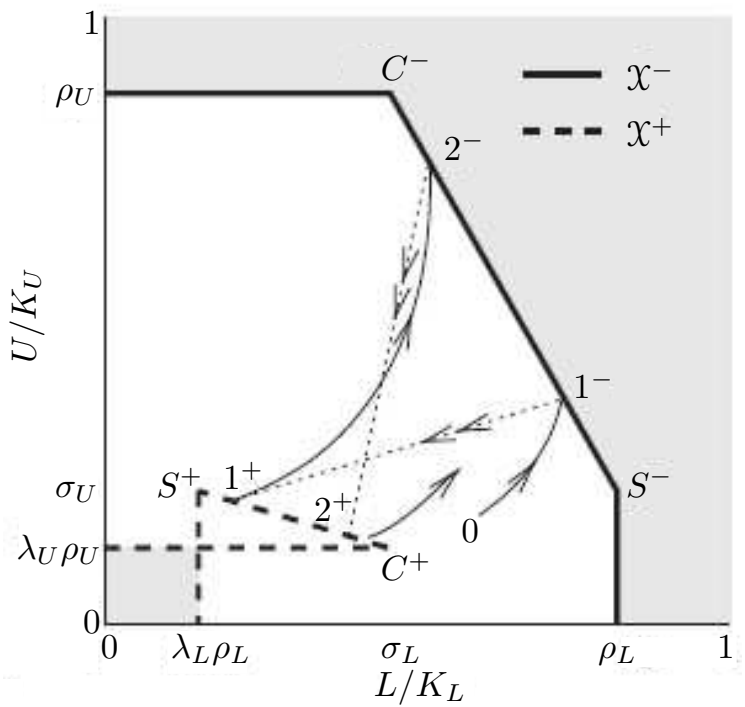

Figure 3: Two-layer model behavior. (a) The pre- and post-fire manifolds $x^{-}$and $X^{+}$; the dotted lines with double arrows are instantaneous transitions from $x^{-}$to $x^{+}$due to a fire; horizontal (vertical) lines correspond to surface (crown) fires; oblique lines starting from the segment $C^{-} S^{-}$of $X^{-}$correspond to mixed fires. (b) State portrait of the model; continuous lines with a single arrow represent the growing phase of the forest and are described by eq. (2). 


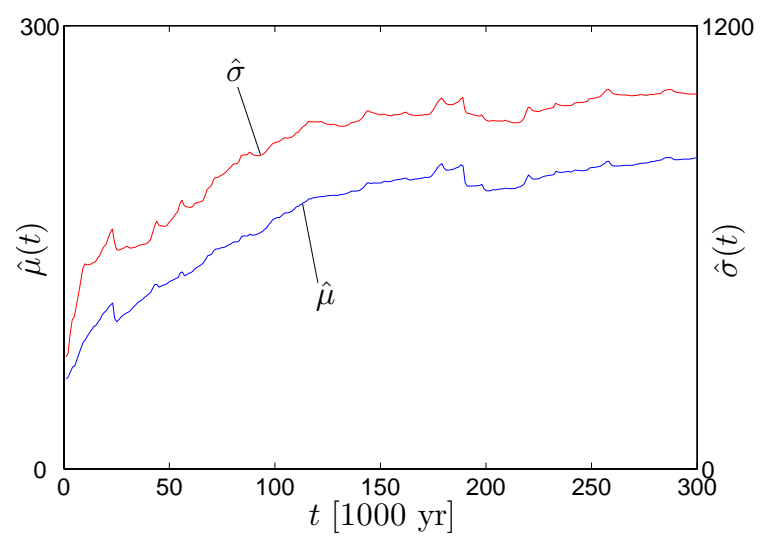

Figure 4: Estimate $\hat{\mu}$ and $\hat{\sigma}$ of the mean and standard deviation of the burned areas as a function of the observation time for the model with $\varepsilon=$ $0.08, r_{L}=3 / 8, r_{U}=1 / 16, K_{L}=K_{U}=1, \alpha=129 / 800, \rho_{L}=0.85, \rho_{U}=$ $14 / 15, \sigma_{L}=0.6, \sigma_{U}=0.35, \lambda_{L}=\lambda_{U}=10^{-4}$. 

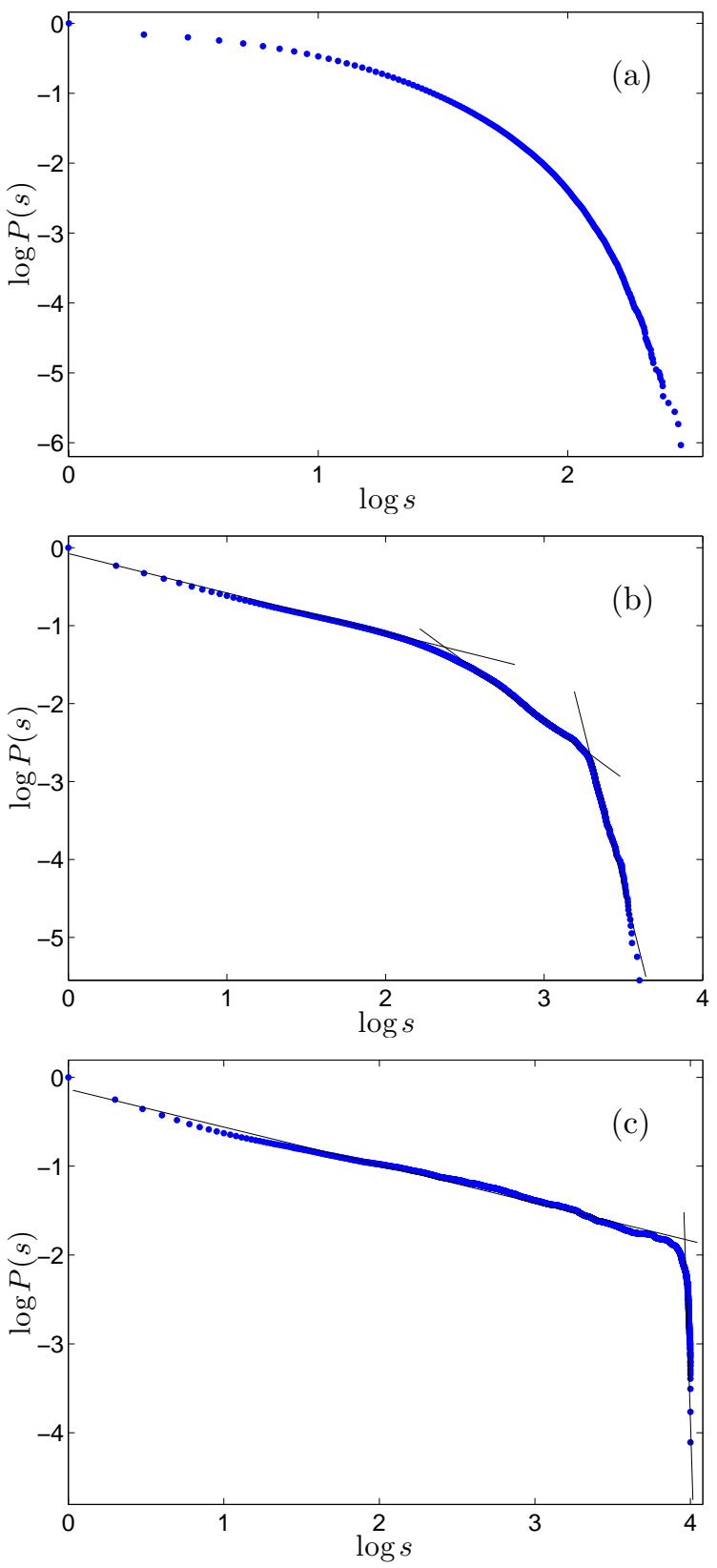

Figure 5: Three examples of cumulative distributions $P(s)=$ prob $[$ burned area $\geq s]$ obtained from the model for different parameter values: (a): $\varepsilon=0.06$; (b): $\varepsilon=0.07$; (c): $\varepsilon=0.08$. Other parameter values as specified in the caption of Fig. 4. 

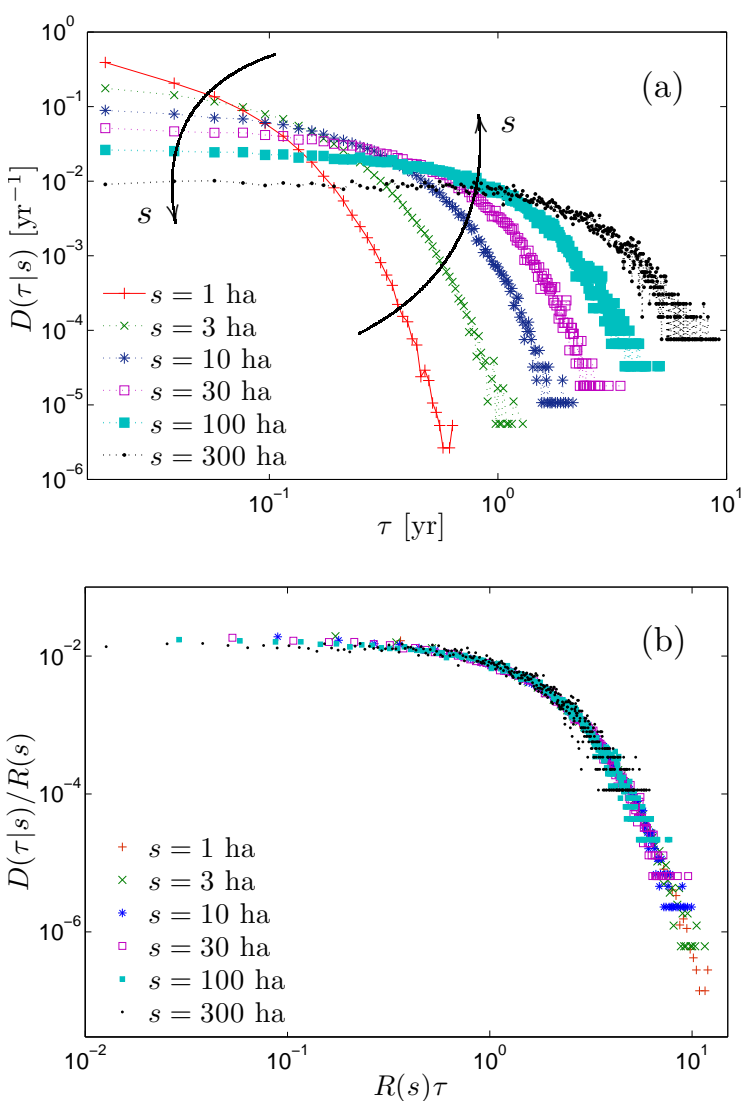

Figure 6: Results of the analysis of the time intervals $\tau$ separating successive fires generated by the model with parameter values as specified in the caption of Fig. 4. (a) Probability densities $D(\tau \mid s)$ for minimum burned areas $s$. (b) The previous densities after rescaling by the mean fire rate $R(s)$. 